\title{
Direct detection of heteroresistance in Mycobacterium tuberculosis using molecular techniques
}

The risk of resistance emerging during the treatment of tuberculosis (TB) is ever present. The simultaneous detection of both ethambutol-sensitive and -resistant genotypes in sputum specimens predicted or known to have drug-resistant TB has been reported, a phenomenon described as heteroresistance (Rinder et al., 2001a). We extend this observation by detecting pre-existing mutants in the sputum of patients from a population in Tanzania in which resistance is unlikely.

Two samples of sputum, either freshly collected (six samples) or frozen (16 samples), from each of 11 newly diagnosed TB patients were examined. DNA extraction was performed at Kilimanjaro Christian Medical College (KCMC), Moshi, Tanzania, using the chelating resin Chelex 100 (Bio-Rad) (Giraffa et al., 2000). The presence of Mycobacterium tuberculosis DNA was confirmed by strand displacement amplification (SDA; BD Probe-Tec) at the Centre for Medical Microbiology, University College London, UK, in nine samples (six fresh and three frozen). Seven frozen samples were equivocal by repeated SDA testing, indicating that the sample DNA had deteriorated on storage (six samples were not tested due to insufficient volume of extract). To detect mutations in codon 306 of the ethambutol-resistance-determining region of $e m b B$, which is responsible for most ethambutol resistance (Sreevatsan et al., 1997), restriction digestion of nested PCR products was carried out as previously described (Rinder et al., 2001b).

Nested $e m b B$ PCR was performed on all 22 samples at KCMC. Twelve extracts gave PCR products of the expected size (103 bp). To identify pre-existent ethambutol mutants, six PCR-positive DNA extracts from three patients were selected, on which nested $e m b B$ PCR was performed 10 times under identical conditions. The PCR product was digested with Nla III (New England Biolabs), which cut the PCR products at three sites, including codon 306 of the wild-type gene, producing four fragments of $11,18,30$ and $44 \mathrm{bp}$ in length. Mutation at codon 306, which indicated the presence of a resistant gene, would be detected by the presence of a 74 bp product $(30+44$ bp due to loss of a restriction site). The restriction products were visualized by electophoresis in a $3 \%$ agarose gel containing ethidium bromide (Fig. 1). All six samples demonstrated the presence of the wild-type sequence in each of the 10 identical tests. However, two samples from one patient and one sample from a second patient demonstrated an additional $74 \mathrm{bp}$ band in at least one of the 10 tests, indicating the presence of a mutation at codon 306 of the embB gene.

Identification of mutations encoding ethambutol resistance in samples from two of three patients indicates that pre-existent mutants are common, even when the risk of drug resistance is low, and that the molecular heterogeneity described previously also occurs in patients at low risk of resistance. Our data demonstrate the ever-present risk of the emergence of resistance if therapy is less than optimal. Due to the possibility of preferential amplification of low levels of a mutant sequence, our data indicate that there is a risk of false-positive results in rapid ethambutol-resistance testing by a nucleic acid amplification method. This may have clinical implications for the initial choice of anti-TB therapy if used to predict phenotypic susceptibility.

\section{Acknowledgements}

The authors would like to acknowledge financial support received for M. M. C. from the Association of Medical Microbiologists.
Fig. 1. Demonstration of both wild-type and mutant restriction products in one out of 10 identical embB PCR reactions (sample: patient 10/day 5). 


\section{Mairi M. Cullen, ${ }^{1}$ Noel E. Sam, ${ }^{2}$ Esther G. Kanduma, ${ }^{3}$ Timothy D. McHugh ${ }^{4}$ and Stephen H. Gillespie ${ }^{4}$}

${ }^{1}$ Department of Microbiology, Wythenshawe Hospital, Southmoor Road, Manchester M23 9LT, UK

${ }^{2}$ Clinical Laboratory, Kilimanjaro Christian Medical Centre, PO Box 3010, Moshi, Tanzania

${ }^{3}$ Department of Biochemistry, University of Nairobi, PO Box 30197, 00100,

Nairobi, Kenya
${ }^{4}$ Centre for Medical Microbiology, Hampstead Campus, University College London, Rowland Hill Street, London NW3 2PF, UK

Correspondence: Timothy D. McHugh (t.mchugh@medsch.ucl.ac.uk)

Giraffa, G., Rossetti, L. \& Neviani, E. (2000). An evaluation of chelex-based DNA purification protocols for the typing of lactic acid bacteria. J Microbiol Methods 42, 175-184.

Rinder, H., Mieskes, K. T. \& Löscher, T. (2001a). Heteroresistance in Mycobacterium tuberculosis. Int J Tuberc Lung Dis 5, 339-345.
Rinder, H., Mieskes, K. T., Tortoli, E., Richter, E., Casal, M., Vaquero, M., Cambau, E.,

Feldmann, K. \& Loscher, T. (2001b).

Detection of $e m b B$ codon 306 mutations in ethambutol resistant Mycobacterium tuberculosis directly from sputum samples: a low-cost, rapid approach. Mol Cell Probes 15, $37-42$.

Sreevatsan, S., Stockbauer, K. E., Pan, X., Kreiswirth, B. N., Moghazeh, S. L., Jacobs, W. R., Jr, Telenti, A. \& Musser, J. M. (1997). Ethambutol resistance in Mycobacterium tuberculosis: critical role of embB mutations. Antimicrob Agents Chemother 41, 1677-1681. 